\title{
Flexibilität in logistischen Systemen
}

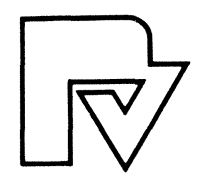


Martin Kühn


Mit 29 Abbildungen

Physica-Verlag Heidelberg 


\section{Reihenherausgeber \\ Prof. Dr. Christoph Schneeweiß, Universität Mannheim}

\section{Autor \\ Dr. Martin Alexander Kühn, Lehrstuhl für Allgemeine Betriebswirtschafts- lehre und Unternehmensforschung, Universität Mannheim, Schloß, D-6800 Mannheim 1}

ISBN-13: 978-3-7908-0450-8

e-ISBN-13: 978-3-642-95884-7

DOI: 10.1007/ 978-3-642-95884-7

Dieses Werk ist urheberrechtlich geschützt. Die dadurch begründeten Rechte, insbesondere die der Übersetzung, des Nachdruckes, des Vortrags, der Entnahme von Abbildungen und Tabellen, der Funksendungen, der Mikroverfilmung oder der Vervielfältigung auf anderen Wegen und der Speicherung in Datenverarbeitungsanlagen, bleiben, auch bei nur auszugsweiser Verwertung, vorbehalten. Eine Vervielfältigung dieses Werkes oder von Teilen dieses Werkes ist auch im Einzelfall nur in den Grenzen der gesetzlichen Bestimmungen des Urheberrechtsgesetzes der Bundesrepublik Deutschland vom 9. September 1965 in der Fassung vom 24. Juni 1985 zulässig. Sie ist grundsätzlich vergütungspflichtig. Zuwiderhandlungen unterliegen den Strafbestimmungen des Urheberrechtsgesetzes.

(C) Physica-Verlag Heidelberg 1989

Die Wiedergabe von Gebrauchsnamen, Handelsnamen, Warenbezeichnungen usw. in diesem Werk berechtigt auch ohne besondere Kennzeichnung nicht zu der Annahme, daß solche Namen im Sinne der Warenzeichen- und Markenschutz-Gesetzgebung als frei zu betrachten wären und daher von jedermann benutzt werden dürften. 
Für Agnes 


\section{G E I E I T W O R T}

Mit der Untersuchung der Flexibilität in logistischen Systemen greift die vorliegende Schrift ein gleichermaßen anspruchsvolles wie hoch aktuelles Thema auf. Flexiblität ist heute von zentraler Bedeutung nicht nur für die Praxis, sondern auch für die Weiterentwicklung einer theoretischen Betriebswirtschaftslehre.

Bei der quantitativen Untersuchung des Flexibilitätsaspektes betrieblicher Entscheidungen stößt man sehr bald auf erhebliche Schwierigkeiten. Zwar gibt es in der Literatur eine Fülle von Definitionen, doch hält keine sämtlichen Erfordernissen an ein derartiges Maß stand. Hier eine allgemein zufriedenstellende Operationalisierung gefunden $\mathrm{zu}$ haben, ist als großes Verdienst des Verfassers zu werten. Dies ist jedoch keineswegs der einzige innovative Beitrag der vorliegenden Schrift. Die Frage nach der Flexibilität logistischer Systeme führt den Autor auf die Untersuchung komplexer, stochastischer, dynamischer Modelle, für die man international bisher noch keine Lösungen hat auffinden können. Durch die geschickte Kombination flexibler Planung und hierarchisch strukturierter Heuristiken ist es dem Autor jedoch gelungen, umfassende Einsichten in die Zusammenhänge zwischen Flexibilität, Informationsstand und Reaktionsfähigkeit dieser für die Praxis wichtigen Systeme offenzulegen.

Besondere Bedeutung kommt hierbei der hierarchischen Planung von Flexibilitätspotentialen zu. Eine solche Planung geschieht über ein Entscheidungsbaumverfahren, dessen erste Entscheidung im strategischen Aufbau eines Flexibilitätspotentiales besteht, das sodann in den nachfolgenden operativen Entscheidungen optimal genutzt wird. Dabei kommt es wesentlich darauf an, das logistische System so auszulegen, daß es in der Lage ist, unvorgesehenen störungen bestmög- 
lich zu begegnen, d.h. es sollte eine hohe Flexibilität aufweisen.

Flexibilität ist hierbei jedoch nicht die einzige Eigenschaft, die es zu beachten gilt. Auch die Robustheit und Elastizität eines logistischen Systems sowie weitere Erfolgskriterien sind von Bedeutung. Sie werden in einen mehrkriteriellen Zusammenhang gebracht und dem Entscheidungsträger als zentrale Beurteilungsgrößen vorgelegt.

Es ist das Verdienst des Autors, hierbei den zentralen Begriff der Flexibilität einer quantitativen Betrachtung zugänglich gemacht zu haben. Es wäre zu wünschen, wenn dieser Begriff nicht nur in logistischen Systemen, sondern auch in anderen Bereichen der Betriebswirtschaftslehre eine anschauliche Bedeutung erhielte.

Mannheim, im Juli 1989

Ch. Schneeweiß 


\section{NHALTSVERZEICHNIS}

1 EINLEITUNG 1

1.1 Ausgangspunkt der Untersuchung 1

1.2 Die Planung von Flexibilitätspotentialen 3

1.3 Generelle Zielsetzung und Gang der Untersuchung 6

2 DIE PLANUNG IN DER UNTERNEHMUNG ALS FLEXIBLES ENTSCHEIDUNGSPROBLEM

2.1 Das betrachtete Modell eines betrieblichen Systems

2.1.1 Das allgemeine Modell A

2.1.2 Die Kombination von Präventiv- und Sanierungsmaßnahmen in der Lagerhaltung: Modell L

2.1.3 Ein Lagerhaltungsmodell mit anpassungsfähiger Systemtechnologie: Modell LAT

2.2 Optimale und effiziente Strategien zur Nutzung einer Technologie

2.2.1 Die optimale Planungsmethode in Modell A: Flexible Planung

2.2.2 Flexible Planung zur Ermittlung effizienter strategien bei mehrfacher zielsetzung

2.2.3 Ein Beispiel zur flexiblen Planung

2.2.3.1 Optimierung eines einzigen Kriteriums

2.2.3.2 Ermittlung effizienter Strategien bei mehrfacher zielsetzung

2.2.4 Die Ermittlung einer Teilmenge effizienter Strategien durch Einführung einer künstlichen Zielfunktion 
2.3 Die Leistungsfähigkeit flexibler Planung

2.3.1 Vergleich mit prophetischer Planung und völligem Planungsverzicht

2.3.2 Myopische versus flexible Planung

52

2.3.3 Starre versus flexible Planung

3.1 Das Phänomen der Flexibilität

3.1.1 Notwendige Anpassungsfähigkeit betrieblicher systeme

3.1.2 Flexibilität und Elastizität

3.2 Ansätze zur Definition und Messung der Flexibilität

3.2.1 Elastizitätsorientierte Ansätze

3.2.2 Robustheitsorientierte Ansätze

67

3.2.3 Zielerreichungsorientierte Ansätze

3.2.3.1 Der Ansatz von Jakob

3.2.3.2 Der Ansatz von Hanssmann

3.3 Ein Konzept zur Operationalisierung des Begriffes Flexibilität

3.3.1 Der Fall eines einzigen Optimierungskriteriums

3.3.1.1 Definition der Flexibilität

3.3.1.2 Ein Beispiel zur Flexibilitätsdefinition

3.3.2 Flexibilität und Robustheit bei mehr-

facher Zielsetzung

3.3.2.1 Flexibilität einer Technologie bei mehrfacher zielsetzung

3.3.2.2 Robustheit einer Strategie

3.3.2.3 Das Kostenkriterium bei mehrfacher zielsetzung

3.3.2.4 Ein Beispiel

3.4 Der Nutzen des flexibilitätsorientierten Planungsansatzes 


\section{DIE FLEXIBILISIERUNG LOGISTISCHER SYSTEME DURCH} SANIERUNGSPOTENTIALE

4.1 Modell LAT2 94

4.1.1 Zweck der Analyse von Modell LAT2 94

4.1.2 Modellbeschreibung 95

4.1.3 Die Formulierung des Modells LAT2 als Markoffscher Entscheidungsprozeß 100

4.1.4 Entscheidungsiteration zur Bestimmung einer optimalen Strategie 103

4.1.5 Effiziente Strategien bei mehrfacher $\begin{array}{ll}\text { Zielsetzung } & 108\end{array}$

4.2 Flexibilität im Modell LAT2 109

$\begin{array}{ll}4.2 .1 \text { Versuchsaufbau } & 109\end{array}$

4.2.2 Untersuchungen zur Flexibilität alternativer Potentiale 112

4.2.2.1 Flexibilität bei einfacher zielsetzung 112

4.2.2.2 Flexibilität bei mehrfacher zielsetzung 117

4.2.3 Strategien zur Nutzung vorhandener Flexibilität $\quad 125$

4.2.3.1 Kostenminimierende Strategien 125

4.2.3.2 Effiziente Strategien bei mehrfacher zielsetzung

\section{PLANUNGSMODELLE ZUR NUTZUNG DER FLEXIBILITÄT} KOMBINIERTER EIL- UND NORMAIMASSNAHMEN

5.1 Auf Modell L basierende Entscheidungsprobleme

5.2 Lagerdisposition mit Normal- und Eilbestellungen

5.2.1 Modell LAG

5.2.1.1 Formulierung des Lagerhaltungsmodells 140

5.2.1.2 Beschreibung des Bedarfsprozesses 142 
5.2.2 Die Zerlegung von Modell LAG

5.2.3 Makromodell zur Disposition der Normalbestellungen

5.2.3.1 Abgrenzung des Makromodells

5.2.3.2 Ein (s, s)-Modell zur Disposition im Makromodell

5.2.4 Mikromodell zur Disposition der Eilbestellungen

5.2.4.1 Abgrenzung des Mikromodells

5.2.4.2 Ein (S,S)-Modell zur Disposition im Mikromodell $\left(\mathrm{K}^{\mathrm{e}}=0\right)$

5.2.5 Kopplung von Mikro- und Makromodell

5.2.6 Ein Beispiel zum Mikro-Makromodell

5.2.7 Anpassung des Modells an sporadische Bedarfe

5.2.7.1 Auswahl einer geeigneten Standardbedarfsverteilung

5.2.7.2 Anpassung des Bestellmengenmodells an sporadische Bedarfe

5.2.8 Anpassung des Modells an fixe Eilbestellkosten $\mathrm{K}^{\mathrm{e}}>0$

5.2.8.1 Disposition der Eilbestellung im Mikromodell

5.2.8.2 Ein Beispiel zur Disposition der Eilbestellung

5.2.8.3 Der Fall mehrerer Eilbestellungen in einem Normalbestellzyklus

5.2.8.4 Anpassung des Lenkfehlmengenkostensatzes

5.3 Kapazitätsplanung: Kombination von regulärer Arbeitszeit und Überstunden

5.3.1 Problemformulierung

5.3.2 Die Anpassung des Mikro-Makromodells an das Kapazitätsplanungsproblem

5.3.3 Optimale flexible Lösung und starre Lösungen des Kapazitätsplanungsproblems 
5.3.4 Ein Zahlenbeispiel zur Kapazitätsplanung 203

5.3.5 Ein numerischer Vergleich zwischen MikroMakromodell, flexibler und starrer Planung

5.4 Die Flexibilisierung eines Systems aufgrund eines Sanierungspotentials

6 DIE BEWALTTGUNG VON UNSICHERHEIT IN DER BETRIEBLICHEN PLANUNG

6.1 Eine allgemeine Beschreibung des betriebswirtschaftlichen Modellbildungsprozesses

6.2 Strategische Planung

6.2.1 Konstruktion hierarchisch strukturierter Untermodelle

6.2.2 Die Auswahl eines Potentials

6.3 Operative Planung

6.3.1 Analyse der Teiluntermodelle bei gegebenem Potential

6.3.2 Konstruktion flexibilitätsorientierter Teiluntermodelle

6.4 Schlußbemerkung 